\section{Super rat prepares to take on obesity research}

Indian scientists have bred a new strain of rat that they believe could upset the lucrative world market in animal models for diabetes and obesity research. The rat can grow as large as 1.4 kilograms - almost five times the size of the average rat - and its breeders think it may have advantages over the obese mutant rats used in laboratories in the West.

The rat, bred at India's National Institute of Nutrition in Hyderabad, has begun to attract international interest. "The NIN rats could potentially be very important animals," says Rudolph Leibel, an obesity researcher at Rockefeller University in New York. Erik Änggard, research director of the William Harvey Research Institute in London, has already asked for some of the animals for his work on non-insulin-dependent diabetes and insulin resistance.

From the age of five weeks, the rat gains weight at a rate of 5 grams per day on average and, at its biggest, is thought to be the fattest mutant rat in the world. So far, no one knows the nature of the mutation responsible. But Nappan Veettil Giridharan, senior research officer for the NIN's Laboratory Animals Information Service Centre, claims that the new strain could be a better alternative to the two most widely used obese rat models, the Zucker and the Koletsky. "What we have

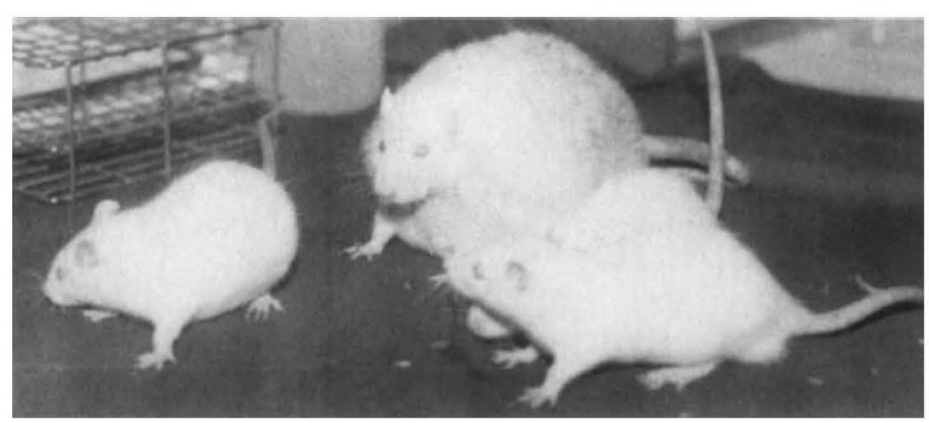

World

heavyweight: the Indian rat may have advantages over its Western counterparts

is a new strain that coulJd have advantages over Western obese models due to its genetic purity and the relative ease with which it can be propagated," he says.

Both the Zucker and Koletsky strains owe their obesity to a mutation in the gene $f a$, for "fatty", which has been localized but not yet cloned. Leibel says the mutation responsible for the NIN rats' obesity needs to be mapped and cloned before the rat's advantages can be confirmed. But he is keen to help and has contacted the NIN to offer to collaborate.

The obese NIN mutant first appeared spontaneously four years ago in a stock of Wistar rats that the institute had maintained since 1920 through inbreeding. Researchers at the institute selectively bred it to establish an obese colony of more than 400 rats, designated WNIN-ob.

In practical terms, what sets the NIN rats apart from other obese models is their unique kinky tails - the fatter the animal, the kinkier the tail - that help to identify carriers of the mutation. While the obese rats all have kinky tails, het- erozygote carriers of the mutation, which are not obese, also have a kinky tail. Obese animals are infertile, but the NIN colony can be easily maintained by mating the kinky-tailed carriers. Zucker rats, by contrast, have no such identifying feature in early life, and must also be outbred with other strains to produce obese offspring.

"While it took us just 3 years to raise 400 obese rats, producing a similar number of Zucker rats would take 12 years," says Giridharan. He also believes that the genetic purity of the stock should hasten the search for the mutation responsible for making the animals obese.

Zucker and Koletsky animals are bred and marketed by only a handful of companies worldwide and the animals do not come cheap. For example, Harlan-Oleac UK, a company based in Huntingdon, England, charges $£ 33$ (\$54) each for Zucker rats at 3 weeks old, and the price rises with their age. The Indian rat would be a lot cheaper, says Giridharan.

K. S. JAYARAMAN New Delhi

\title{
Transplants legal at last - but not necessarily practicable
}

Surgeons in Japan are finally free to perform heart and liver transplants, almost 30 years after Japan's first and only heart transplant operation ended in demands for a murder trial. A bill passed by the parliament's upper house sets out a legal framework for organ transplants, and concludes a tortuously protracted debate about whether organs can legally be harvested for such transplants from "braindead" bodies.

Under the legislation, organs can be harvested following brain death "in instances where the donor has provided in advance a written declaration of intent and there is no opposition from the family". If no such permission exists, the patient's heart will have to stop beating before death can be declared. This awkward compromise highlights the difficulty that Japan has experienced in coming to terms with the idea of brain death.

Surgeons in Japan fear that these strict rules may make it practically very difficult to perform transplants as very few hearts and livers are likely to become available (Nature Medicine 3, 598, 1997).

RICHARD NATHAN

Tokyo
Appointments ...

Dr. John Coffin (Tufts University School of Medicine) has been appointed Director of a new National Institutes of Health, National Cancer Institute program on HIV research. Coffin, an expert on retroviruses, will coordinate the NCI's retrovirus and HIV-related research and establish a program to investigate retrovirus diversity and HIV drug resistance.

and announcements

The Hereditary Disease Foundation, the organization that coordinated the successful mapping, identification and characterization of the Huntington's Disease gene, has announced the launch of a new project - The Cure HD Initiative, which will coordinate efforts to discover a therapy for HD. - A.J.I. 